\title{
Real-world outcomes of chemo-antiangiogenesis versus chemo-immunotherapy combinations in EGFR-mutant advanced non-small cell lung cancer patients after failure of EGFR-TKI therapy
}

\author{
Xin Yu ${ }^{1 \#}$, Jiaqi Li ${ }^{2 \#}$, Lingyun Ye ${ }^{1 \#}$, Jing Zhao ${ }^{1}$, Mengqing Xie ${ }^{1}$, Juan Zhou ${ }^{1}$, Yinchen Shen ${ }^{2}$, Fei Zhou ${ }^{1}$, \\ Yan $\mathrm{Wu}^{1}$, Chaonan $\mathrm{Han}^{1}$, Jialin Qian', Tianqing $\mathrm{Chu}^{2}$, Chunxia Su${ }^{1}$ \\ ${ }^{1}$ Department of Oncology, Shanghai Pulmonary Hospital \& Thoracic Cancer Institute, Tongji University School of Medicine, Shanghai, China; \\ ${ }^{2}$ Department of Pulmonary Medicine, Shanghai Chest Hospital, Shanghai Jiao Tong University, Shanghai, China \\ Contributions: (I) Conception and design: C Su, T Chu; (II) Administrative support: None; (III) Provision of study materials or patients: None; \\ (IV) Collection and assembly of data: J Zhao, M Xie, J Zhou, Y Shen, F Zhou, Y Wu, C Han, J Qian; (V) Data analysis and interpretation: X Yu, J Li, \\ L Ye; (VI) Manuscript writing: All authors; (VII) Final approval of manuscript: All authors. \\ "These authors contributed equally to this work. \\ Correspondence to: Chunxia Su. Department of Oncology, Shanghai Pulmonary Hospital \& Thoracic Cancer Institute, Tongji University School of \\ Medicine, Shanghai, China. Email: susu_mail@126.com; Tianqing Chu. Department of Pulmonary Medicine, Shanghai Chest Hospital, Shanghai \\ Jiao Tong University, Shanghai, China. Email: tianqing_chu@126.com.
}

Background: Despite the potent efficacy of epidermal growth factor receptor (EGFR)-tyrosine kinase inhibitors (TKIs) in the treatment of EGFR-mutant non-small cell lung cancer (NSCLC) patients, drug resistance inevitably ensues, and there remains a paucity of treatment options in clinical practice.

Methods: We identified patients with EGFR-mutant advanced NSCLC presenting to Shanghai Pulmonary Hospital and Shanghai Chest Hospital between January 2015 and December 2020 treated with chemoantiangiogenesis or chemo-immunotherapy combinations after EGFR-TKI resistance. Patient information was collected, and the objective response rate (ORR), disease control rate (DCR), and progression-free survival (PFS) were assessed.

Results: A total of 144 patients who met our inclusion criteria were enrolled. Chemo-immunotherapy combinations achieved a higher objective response rate (ORR) compared with chemo-antiangiogenesis combinations (29.5\% vs. 13.0\%, $\mathrm{P}=0.018)$. The DCR was similar between the two groups $(93.0 \%$ vs. $88.6 \%$, $\mathrm{P}=0.585)$, as was the median PFS (7.59 vs. 6.90 months, $\mathrm{P}=0.552)$. In the subgroup analyses, patients who developed secondary T790M mutations after EGFR-TKI treatment were less likely to benefit from chemoimmunotherapy combinations than their T790M-negative counterparts (3.42 vs. 7.63 months, $\mathrm{P}=0.028$ ). For patients who received chemo-antiangiogenesis combinations after TKI resistance, no significant difference was observed in the median PFS between T790M-positive and T790M-negative patients (median PFS: 5.33 vs. 7.46 months, $\mathrm{P}=0.202$ ). Additionally, multivariate analysis showed that an elevated platelet count was independently associated with a worse PFS for both groups.

Conclusions: The efficacy of chemo-immunotherapy combinations was comparable to chemoantiangiogenesis combinations after failure of EGFR-TKI therapy. For patients harboring EGFR T790M mutations, chemo-antiangiogenesis combinations may be the preferred therapeutic option. In addition, platelet count could be a potential prognostic factor for patients after failure of EGFR-TKI therapy. Further research should be conducted on larger populations and in a prospective setting.

Keywords: Non-small cell lung cancer (NSCLC); EGFR-TKIs; antiangiogenic therapy; immunotherapy; resistance 
Submitted Aug 06, 2021. Accepted for publication Sep 15, 2021.

doi: 10.21037/tlcr-21-681

View this article at: https://dx.doi.org/10.21037/tlcr-21-681

\section{Introduction}

In the past decade, multiple oncogenic molecular alterations have been discovered in lung adenocarcinoma (LUAD) that not only contribute to its carcinogenic nature but also serve as potential drug targets for therapy. Somatic mutations of the epidermal growth factor receptor (EGFR) are seen in approximately $15 \%$ of Caucasians and $40-60 \%$ of East Asians with LUAD (1). Several phase III clinical trials in advanced non-small cell lung cancer (NSCLC) patients harboring EGFR mutations have revealed the superior efficacy of EGFR tyrosine kinase inhibitors (TKIs) compared to conventional platinumbased cytotoxic agents (2). Nevertheless, most patients ultimately develop acquired resistance, which remains a major unsolved problem and has required the development of more advanced therapies (3).

Considering the complexity of tumor heterogeneity after TKI resistance, overcoming the specific resistance mechanisms with targeted compounds is of great clinical importance to ensure better outcomes (4). Following EGFR-TKI therapy, the EGFR T790M mutation in exon 20 has emerged as the dominant resistance mechanism to first- and second-generation EGFR-TKIs, resulting in the development of third-generation TKIs. Osimertinib monotherapy is the currently recommended treatment regimen for EGFR T790M-positive patients (5). Other resistance mechanisms of first- and second-generation EGFR-TKIs include bypass pathway activation (e.g., HER2 and MET amplification), downstream signaling pathways (e.g., BRAF and PI3K mutations), histological transformation into small-cell lung cancer (SCLC) and epithelial-mesenchymal transition (EMT) (4). Molecular mechanisms of acquired resistance to third-generation EGFR-TKIs is highly heterogeneous and can be broadly grouped into EGFR-dependent and EGFR-independent mechanisms. However, the resistance mechanisms to osimertinib in roughly half the EGFR-mutant cases remain undiscovered $(6,7)$. To date, multiple preclinical and clinical studies have reported promising results using novel targeted agents to deal with TKI resistance. But there is no standardized care for this scenario, and further systemic treatments are often required for these patients. In clinical practice, most patients would be offered platinum-based doublet chemotherapy with or without the combination of antiangiogenic therapy $(8,9)$.

Advances in immune checkpoint inhibitors (ICIs) targeting programmed cell death protein-1 (PD-1) and programmed cell death ligand-1 (PD-L1) have dramatically changed the treatment landscape for NSCLC, demonstrating significant improvements in overall response and survival compared with standard chemotherapy. Unfortunately, multiple trials and retrospective studies suggest that patients harboring the EGFR mutation who receive ICIs do not achieve a favorable outcome. In fact, early trials showed a trend toward survival detriment in patients treated with ICI monotherapy (10). Further investigation suggested that the combination of TKIs and ICIs or the sequence of TKIs after ICI therapy was associated with a potentially increased risk of toxicity such as pneumonitis and hepatotoxicity, with no improvement in efficacy $(11,12)$. The uninflamed tumor microenvironment (TME) and the low immunogenicity of EGFR-mutant tumors could partially explain the potential mechanisms underlying these poor outcomes $(13,14)$. Nevertheless, some patients who acquire resistance to EGFR-TKIs could benefit from immunotherapy-based combinations. Promising data from the IMpower150 and CT18 study has inspired the addition of immunotherapy and antiangiogenic therapy to chemotherapy as effective salvage therapies in EGFR-mutant patients $(15,16)$. A Chinese retrospective study revealed that patients who received combination immunotherapy seemingly acquired longer PFS than those who received monotherapy after EGFRTKIs resistance (17). These results suggest a potential ongoing role for ICIs in these patients, making further research a high priority.

Given the increasing prevalence of EGFR-affected NSCLC and the lack of therapeutic choices at the time of acquired resistance to EGFR-TKIs, investigations into efficacious therapies and subgroup identification are of vital importance. Therefore, we conducted this two-center study to investigate the efficacy of chemo-antiangiogenesis and chemo-immunotherapy combinations in EGFR-mutant advanced NSCLC patients after EGFR-TKI failure to provide new insights into the management of this patient group. We present the following article in accordance with the STROBE reporting checklist (available at https://dx.doi. 
org/10.21037/tlcr-21-681).

\section{Methods}

\section{Study design}

This two-center retrospective study was conducted to compared the efficacy of chemo-antiangiogenesis versus chemo-immunotherapy combinations after EGFRTKI resistance in patients with EGFR-mutant advanced NSCLC. The medical records of EGFR-mutant advanced NSCLC patients treated with chemo-antiangiogenesis or chemo-immunotherapy combinations after EGFR-TKI resistance in Shanghai Pulmonary Hospital (Shanghai, China) and Shanghai Chest Hospital (Shanghai, China) between January 2015 and December 2020 were reviewed. After EGFR-TKI resistance, patients who had received at least one assessment of response to chemo-antiangiogenesis or chemo-immunotherapy combinations were considered eligible. Patients who were lost to follow-up were excluded from the analysis. Chemo-antiangiogenesis combinations were defined as chemotherapy combined with antiangiogenic therapy. Chemo-immunotherapy combinations were defined as chemotherapy combined with PD-1/PD-L1 inhibitors. The criteria for receiving PD-1/ PD-L1 inhibitors in EGFR-mutant advanced NSCLC patients were as follows: a clinician-assessed potential clinical benefit, a stable performance status, tolerance of treatment, and no need for immediate intervention to treat or prevent serious complications associated with disease progression. All procedures performed in this study involving human participants were in accordance with the Declaration of Helsinki (as revised in 2013). The study was approved by the institutional review board of Shanghai Pulmonary Hospital and Shanghai Chest Hospital. Individual consent for this retrospective analysis was waived.

\section{Patient characteristics}

A total of 144 patients were identified and enrolled in this study. The inclusion criteria were as follows: (I) pathologically or cytologically confirmed NSCLC; (II) unresectable stage IIIB/IV according to the eighth edition of the TNM classification for lung cancer; (III) confirmed EGFR mutations detected by next-generation sequencing (NGS) or amplification-refractory mutation system polymerase chain reaction (ARMS-PCR); (IV) the receipt of EGFR-TKI treatment and confirmed disease progression using radiological examinations, including chest computed tomography (CT), brain magnetic resonance imaging (MRI), positron emission tomography (PET), bone scans, and ultrasound examination or CT of the abdomen; (V) the receipt of chemo-antiangiogenesis or chemo-immunotherapy combinations after EGFR-TKI resistance; (VI) at least one measurable lesion according to the Response Evaluation Criteria in Solid Tumors v1.1 (RECIST); (VII) An Eastern Cooperative Oncology Group performance status (ECOG PS) score of 0-2; (VIII) sufficient organ functioning. The exclusion criteria were as follows: (I) other driver gene mutations, including ALK/ROS1 rearrangements and BRAF mutations; (II) an ongoing response with EGFR-TKI treatment; (III) incomplete radiological records and images; (IV) interruption of treatment for intolerable toxicity or nonmedication reasons. All cases met the inclusion and exclusion criteria were carefully identified by experienced thoracic oncologists.

\section{Efficacy assessment and follow-up}

Tumor response was assessed using RECIST v1.1. The first disease response assessment was performed at the completion of two cycles of treatment. The ORR analysis was based on best overall response (BOR), as defined by the percentage of patients achieving partial response or complete response. The disease control rate (DCR) was defined as the rate of partial response, complete response, and stable disease. PFS was defined as the time from the start of chemoantiangiogenesis or chemo-immunotherapy combinations to disease progression. The data cutoff was April 2021, and patients with an ongoing response at this time or the last date of follow-up were considered as censored.

\section{Statistical analyses}

The Mann-Whitney $U$ test was used for continuous variables and Fisher's exact test or Pearson's $\chi^{2}$ for categorical variables. PFS was estimated using the KaplanMeier method, and the log-rank test was used to assess survival difference between two groups. All tests were twosided and a $\mathrm{P}$ value of $<0.05$ was considered statistically significant. The statistical analyses were performed using SPSS version 25.0 (Chicago, IL, USA) and GraphPad Prism version 8.0 (San Diego, CA, USA). The optimal cutoff values of the clinical parameters for PFS were achieved using X-tile software (New Haven, CT, USA) (18). 


\section{Results}

\section{Patient characteristics}

Between January 2015 and December 2020, 144 patients who experienced disease progression following EGFRTKI treatment and who received chemo-antiangiogenesis or chemo-immunotherapy combinations as subsequent therapies were enrolled as previously described with a median follow-up of 8.9 months. The median age of all patients was 61 years (range, 19-76 years), and 52.8\% of patients were female. All patients were pathologically diagnosed with lung adenocarcinoma. A total of 110 (76.4\%) patients were never-smokers. A higher proportion of patients received chemo-antiangiogenesis combinations (100/144, 69.4\%) compared with those who were treated with chemo-immunotherapy combinations (44/144, $30.6 \%)$. The median age of the chemo-antiangiogenesis combination group was higher than that of the chemoimmunotherapy combination group. Other clinical characteristics were balanced between the two groups. The baseline demographic and clinical characteristics of patients are summarized in Table 1.

\section{Efficacy of EGFR-TKIs, and T790M status after EGFR-TKI failure}

Among the 144 patients, 131 (91.0\%) received EGFRTKIs as a single treatment, and $13(9.0 \%)$ received EGFRTKIs in combination with other therapies, including antiangiogenic therapy $(6,4.2 \%)$, radiotherapy (5, $3.5 \%$ ), and chemotherapy (2, 1.4\%). EGFR-TKIs were administered as a first-line treatment to $129(89.6 \%)$ patients, as a second-line treatment to $14(9.7 \%)$ patients, and as a third-line treatment and beyond to $1(0.7 \%)$ patient. For patients who received EGFR-TKIs as second or later-line therapy, EGFR mutations were initially detected by a tissue or blood re-biopsy performed after disease progression on previous therapies. Among all patients, $139(96.5 \%)$ received first-generation EGFR-TKIs, and $5(3.5 \%)$ received second-generation EGFR-TKIs. None were initially treated with third-generation EGFR-TKIs. The ORR and DCR of EGFR-TKIs for all patients were $60.2 \%$ and $93.2 \%$, respectively, and the median PFS was 10.42 months. These results were in accord with the efficacy of first- and second-generation TKIs reported in previous studies. After EGFR-TKI resistance, the T790M secondary mutation was detected in $29(20.1 \%)$ patients. Of them, 23 (79.3\%) patients received third-generation EGFR-TKIs as subsequent therapy. The relatively lower T790M mutation rate in our cohort compared with previous studies could be due to a high rate of false-negative results from the liquid biopsies used for most patients (Table 2).

\section{Efficacy of chemo-antiangiogenesis versus chemo- immunotherapy combinations after EGFR-TKI resistance}

After disease progression following EGFR-TKI treatment, chemo-immunotherapy combinations resulted in a higher objective response rate (ORR) compared with chemo-antiangiogenesis combinations $(29.5 \%$ vs. $13.0 \%$, $\mathrm{P}=0.017$ ), but the DCR was similar between the two groups $(93.0 \%$ vs. $88.6 \%, \mathrm{P}=0.585)$. The median PFS of the chemo-immunotherapy combination group was slightly longer but did not achieve statistical significance (7.59 vs. 6.90 months, $\mathrm{P}=0.552$, HR $=0.875,95 \%$ CI: $0.565-1.355$ ). Overall survival (OS) was premature until the data cutoff. In the subgroup analyses, no significant differences were observed in ORR and PFS in patients with the 19del and L858R mutations who received chemo-antiangiogenesis combinations (ORR: $10.9 \%$ vs. $11.8 \%, \mathrm{P}=1.000$; median PFS: 7.74 vs. 7.30 months, $\mathrm{P}=0.702, \mathrm{HR}=0.920,95 \%$ CI: 0.594-1.426). Furthermore, the ORR and PFS were comparable in T790M-positive and T790M-negative patients after TKI resistance in this group (ORR: 9.5\% vs. $13.2 \%, \mathrm{P}=0.942$; median PFS: 5.33 vs. 7.46 months, $\mathrm{P}=0.202, \mathrm{HR}=1.459,95 \% \mathrm{CI}: 0.737-2.890$ ). For patients who received chemo-immunotherapy combinations, the ORR and PFS were better in patients harboring EGFR L858R mutations than those harboring 19del mutations but showed no significant difference (ORR: $33.3 \%$ vs. $22.7 \%$, $\mathrm{P}=0.438$; median PFS: 7.59 vs. 5.65 months, $\mathrm{P}=0.798$, HR $=0.899$, 95\% CI: 0.392-2.059). However, patients who developed secondary T790M mutations after EGFRTKI treatment were less likely to benefit from chemoimmunotherapy combinations (ORR: $14.3 \%$ vs. $32.4 \%$, $\mathrm{P}=0.608$; median PFS: 3.42 vs. 7.63 months, $\mathrm{P}=0.028$, HR $=3.028,95 \%$ CI: $0.575-15.94$ ) (Figure 1).

\section{Univariate and multivariate analyses of the clinical parameters associated with PFS after EGFR-TKI resistance}

We performed univariate and multivariate analyses to evaluate which clinical parameters were predictors of PFS in the two patient groups after EGFR-TKI resistance. All variables with a $\mathrm{P}$ value $<0.1$ in the univariate analysis were included in the multivariate models. Logistic regression 
Table 1 Baseline demographic and clinical characteristics of patients stratified by treatment strategies after TKI resistance ( $\mathrm{n}=144)$

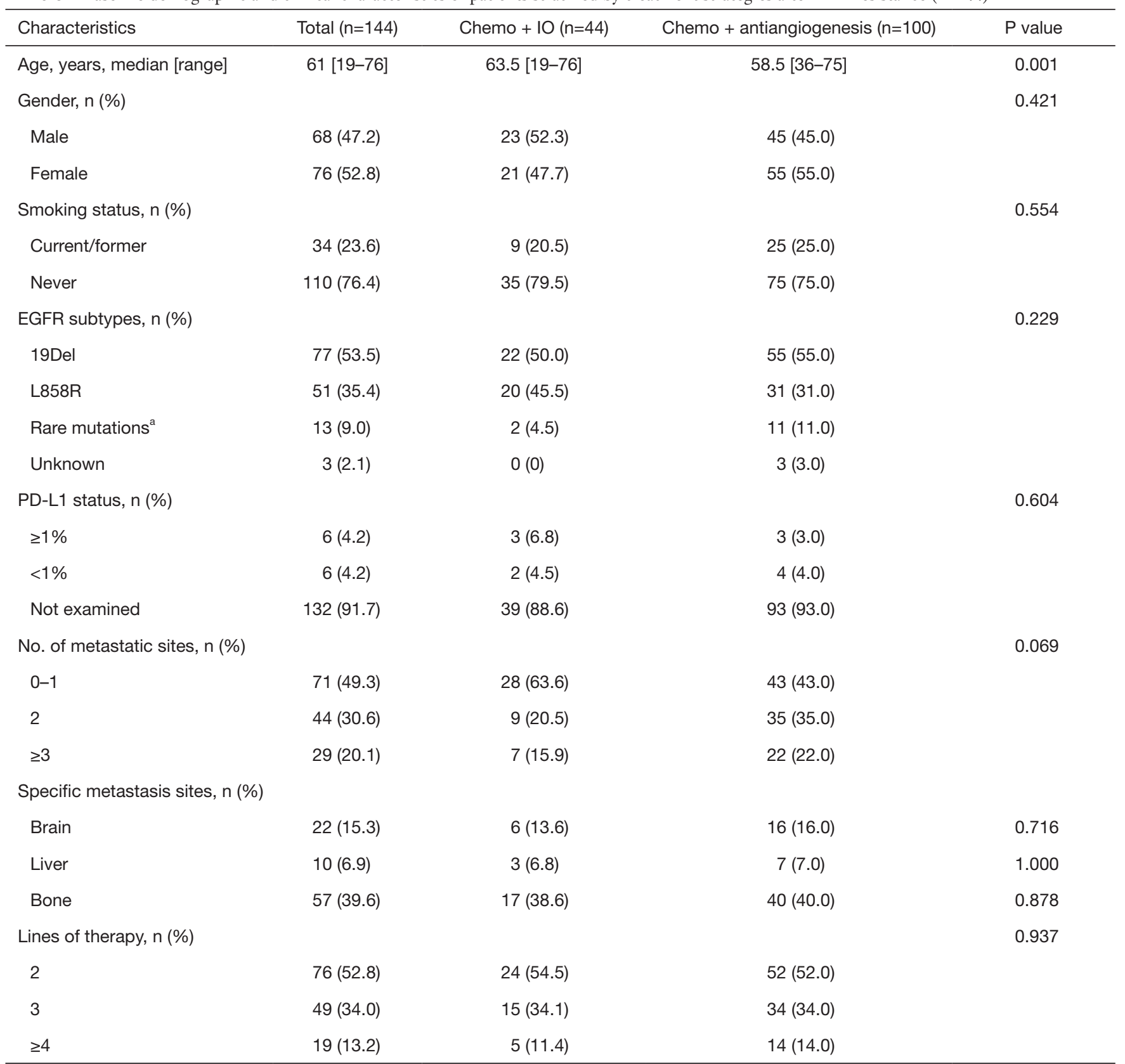

a, rare mutations include four L861Q, three G719X, two Ex20ins, two L858R and EX20ins co-mutations, one L858R and S768I co-mutation, one L858R and L861Q co-mutation. TKIs, tyrosine kinase inhibitors; IO, immunotherapy; EGFR, epidermal growth factor receptor; PD-L1, programmed cell death ligand 1.

models were used to study the effects of variables on outcomes, and X-tile software was applied to calculate the optimal cut-off value of each variable. In the chemoantiangiogenesis combination group, univariate analysis showed that age, gender, median PFS of TKI, lymphocyte count, neutrophil count, monocyte count, eosinophil count, platelet count, and platelet-to-lymphocyte ratio (PLR) had significant effects on PFS. However, multivariate analysis indicated only a platelet count $\leq 373 \times 10^{9} / \mathrm{L}$ was independently associated with a longer PFS $(\mathrm{P}=0.037$, $\mathrm{HR}=0.334,95 \% \mathrm{CI}: 0.119-0.937)$. In the chemoimmunotherapy combination group, univariate analysis 
Table 2 Efficacy of TKIs, T790M status, and efficacy of subsequent therapy of patients ( $\mathrm{n}=144)$

\begin{tabular}{|c|c|c|c|c|}
\hline Characteristics & Total $(n=144)$ & Chemo + IO $(n=44)$ & Chemo + antiangiogenesis $(n=100)$ & $P$ value \\
\hline PR, n (\%) & $71(49.3)$ & $27(61.4)$ & $44(44.0)$ & \\
\hline $\mathrm{SD}, \mathrm{n}(\%)$ & $39(27.1)$ & $11(25.0)$ & $28(28.0)$ & \\
\hline PD, n (\%) & $8(5.6)$ & $2(4.5)$ & $6(6.0)$ & \\
\hline ORR (\%) & 60.2 & 67.5 & 56.4 & 0.244 \\
\hline DCR (\%) & 93.2 & 95.0 & 92.3 & 0.715 \\
\hline Median PFS of TKIs (months) & 10.42 & 10.59 & 10.32 & 0.596 \\
\hline \multicolumn{5}{|l|}{ T790M status after TKIs resistance, $\mathrm{n}(\%)$} \\
\hline $\begin{array}{l}\text { Third-generation TKIs used in T790M positive } \\
\text { patients, } \mathrm{n}(\%)\end{array}$ & $23(79.3)$ & $7(100)$ & $15(68.2)$ & 0.147 \\
\hline \multicolumn{5}{|l|}{ Best response to subsequent treatment } \\
\hline $\mathrm{PR}, \mathrm{n}(\%)$ & $26(18.1)$ & $13(29.5)$ & $13(13.0)$ & \\
\hline $\mathrm{SD}, \mathrm{n}(\%)$ & $106(73.6)$ & $26(59.1)$ & $80(80.0)$ & \\
\hline PD, n (\%) & $12(8.3)$ & 5 (11.4) & $7(7.0)$ & \\
\hline ORR (\%) & 18.1 & 29.5 & 13.0 & 0.017 \\
\hline DCR (\%) & 91.7 & 88.6 & 93.0 & 0.513 \\
\hline
\end{tabular}

a, unknown response was not included in the ORR and DCR analyses. TKIs, tyrosine kinase inhibitors; PR, partial response; SD, stable disease; PD, progressive disease; ORR, objective response rate; PFS, progression-free survival.

showed that T790M status after TKI resistance, lymphocyte count, neutrophil count, eosinophil count, platelet count, neutrophil-to-lymphocyte ratio (NLR), monocyte-tolymphocyte ratio (MLR), and PLR were predictors of PFS. Multivariate analysis also indicated that a platelet count $\leq 264 \times 10^{9} / \mathrm{L}$ was independently associated with a longer PFS $(\mathrm{P}=0.028, \mathrm{HR}=0.256,95 \% \mathrm{CI}: 0.076-0.865)$, which was similar to the conclusion from the chemo-antiangiogenesis combination group (Figure 2; Tables S1,S2). These findings demonstrated that platelet count could be a potential prognostic factor for EGFR-mutant advanced NSCLC patients after disease progression following EGFR-TKI therapy and should be further verified in OS analyses.

\section{Discussion}

Acquired resistance remains a major challenge in the management of oncogene-driven NSCLC. After failure of first-line TKI therapy, the PFS of chemotherapy in second and subsequent lines seems to be substantially lower. The AURA3 study reported a median PFS of 4.4 months with platinum-pemetrexed treatment in a T790M-positive population, and the IMPRESS study reported a median PFS of 5.4 months with platinum-doublet chemotherapy with or without gefitinib $(19,20)$. But few studies have focused on the clinical application of chemotherapy-based combinations after disease progression following EGFR-TKI therapy. Our present study demonstrated that the median PFS of chemo-immunotherapy and chemo-antiangiogenesis combinations were comparable in an unselected patient population and superior to the chemotherapy alone group as previously reported, suggesting both treatments could serve as optional salvage therapies after failure of EGFR-TKIs. This finding is consistent with the results of the subgroup 


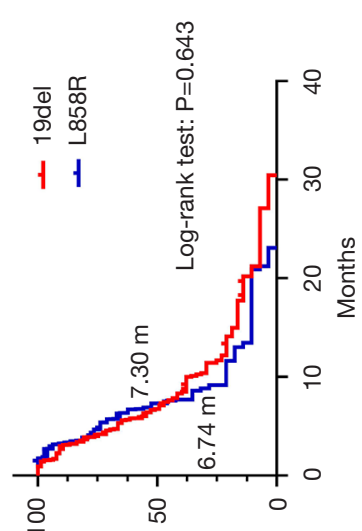

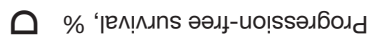
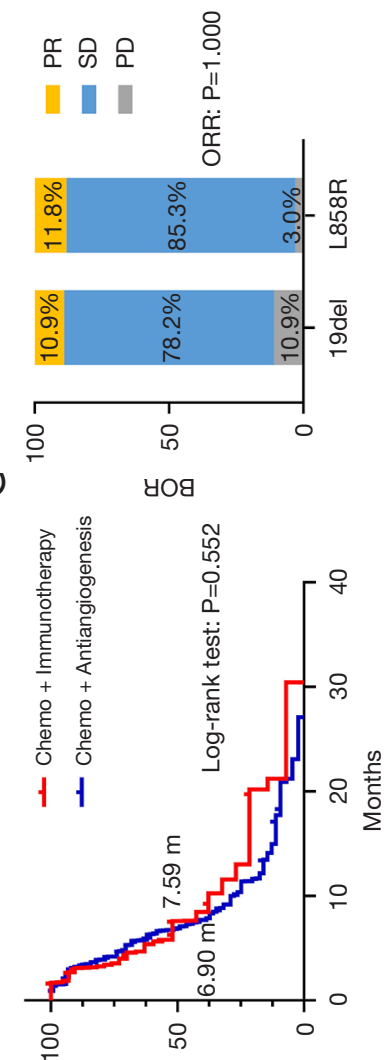

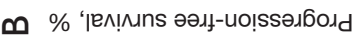

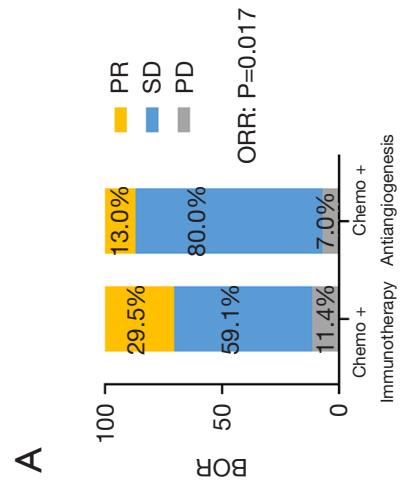

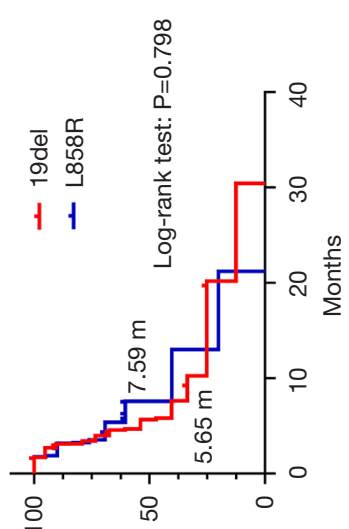

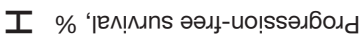

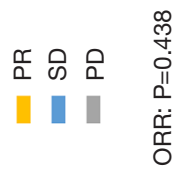

$\checkmark$
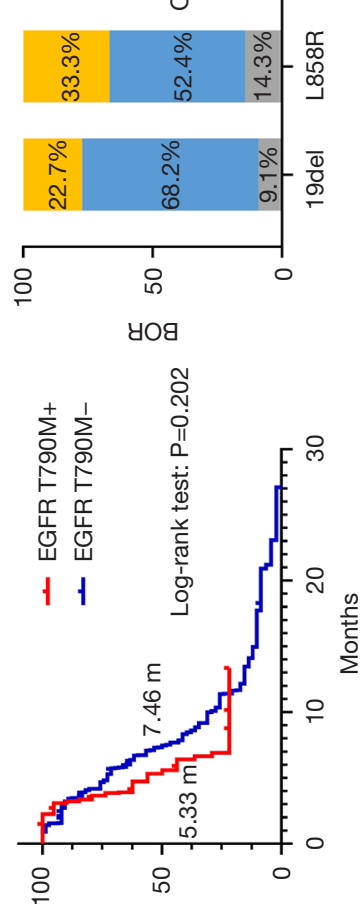

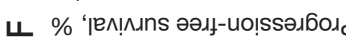
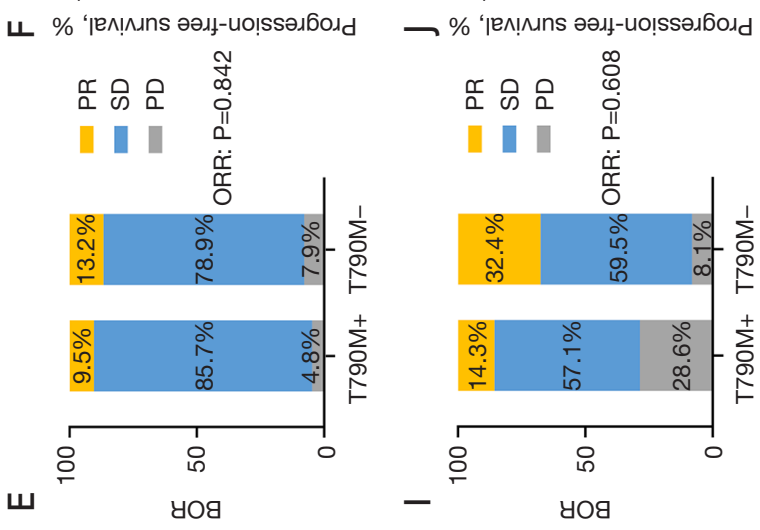

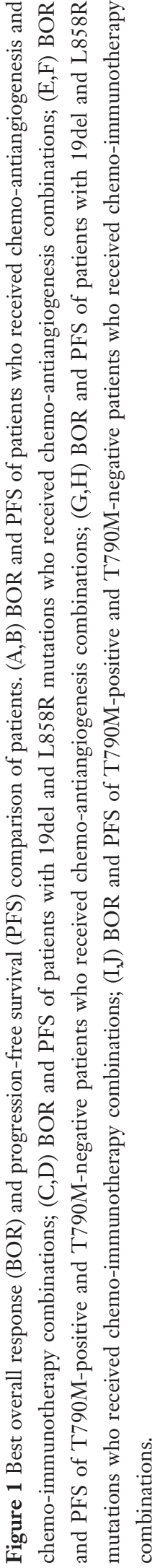


A

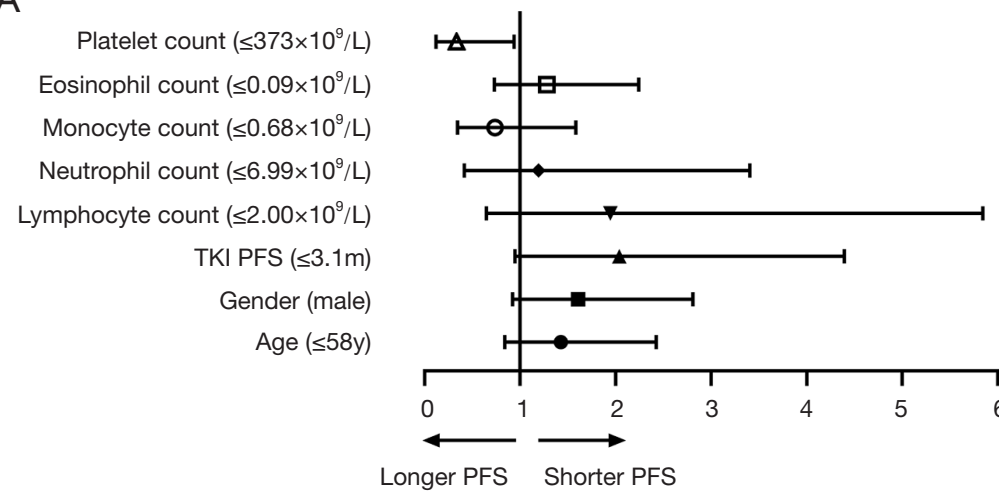

B

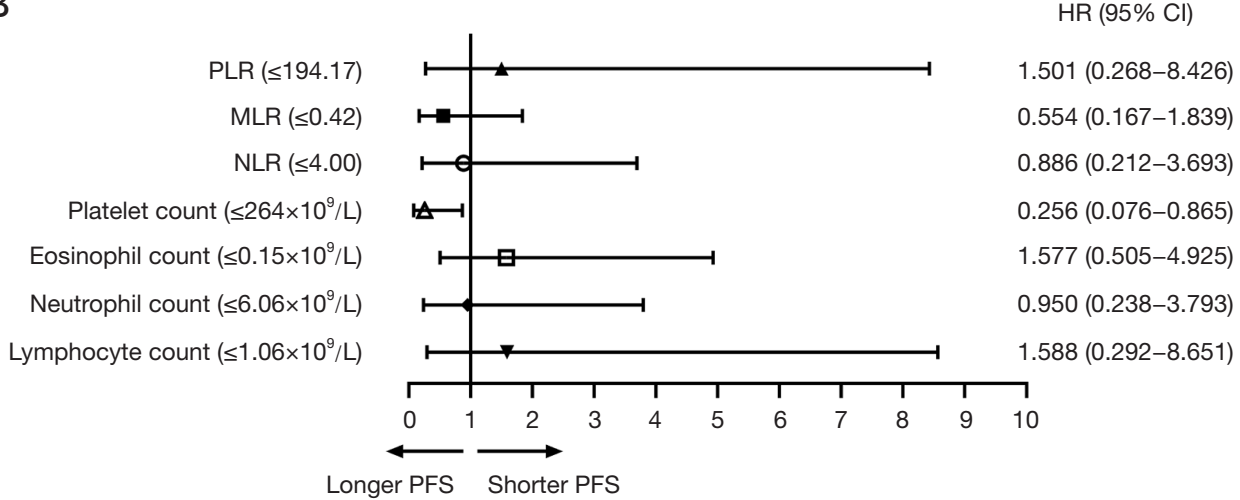

Figure 2 Forest plot of the clinical parameters associated with PFS in subgroup analyses. (A) Forest plot of the multivariate analysis of the clinical parameters associated with PFS in the chemo-antiangiogenesis combination group; (B) forest plot of the multivariate analysis of the clinical parameters associated with PFS in the chemo-immunotherapy combination group.

analysis from the IMpower150 trial, which reported a similar median PFS for atezolizumab plus carboplatin plus paclitaxel (ACP) versus bevacizumab plus carboplatin plus paclitaxel (BCP) treatment in EGFR-positive patients who had previously received TKI therapy (5.7 vs. 6.1 months) (15). In the context of the available literature, this study presents the largest cohort to date to assess the efficacy of chemoimmunotherapy and chemo-antiangiogenesis combinations in TKI-treated advanced NSCLC patients. In addition, the IMpower150 study also illustrated an improved survival for patients treated with atezolizumab plus bevacizumab plus carboplatin plus paclitaxel (ABCP) compared with those given BCP in the subgroup of patients with EGFR mutations. Unfortunately, we only identified two patients in our medical records review who received chemotherapy combined with ICIs and bevacizumab.

A Japanese study demonstrated the superior efficacy of nivolumab in T790M-negative patients with EGFR mutations after EGFR-TKI treatment, possibly due to a higher PD-L1 expression level in this patient group (21). Our study also revealed that the efficacy of chemo-immunotherapy combinations after disease progression following EGFRTKI therapy tended to be greater for T790M-negative than T790M-positive patients. Notably, seven T790M-positive patients in our cohort who received chemo-immunotherapy combinations had received prior osimertinib treatment, suggesting that chemo-immunotherapy combinations were not appropriate therapeutic choices for T790M-positive patients even after progressing on osimertinib. Chemoantiangiogenesis combinations may be the preferred option for these patients. Apart from secondary T790M mutations, a recent study observed better response rates and overall survival outcomes of EGFR L858R tumors than EGFR $19 \mathrm{del}$ cases (22). Another report evaluating the outcomes of 27 patients with EGFR mutations on ICIs found the best response rate in cases with rare EGFR alterations such as G719X and exon 20 insertions (23). These phenomena highlight potential differences between EGFR alleles in response to immunotherapy.

Except for the molecularly heterogeneous features of 
EGFR mutations, other factors are also thought to be responsible for the efficacy of ICIs in EGFR-mutant lung cancers. A retrospective study demonstrated that patients who responded to prior EGFR-TKIs for $>10$ months exhibited a significantly shorter response to ICIs than those who had responded for $\leq 10$ months (24). Su et al. (25) discovered that patients with a poor response to EGFR-TKIs, especially those with de novo resistance, exhibited a high level of immunogenicity, suggesting that these patients may benefit from immunotherapy. However, similar trends were not found in the present study. Results from an Italian cohort suggested a potentially different benefit between former or current smokers and never-smokers, likely due to a different tumor mutational burden (TMB) or PD-L1 expression (26). Therefore, a greater understanding of the TME and further research on the predictive biomarkers of immunotherapy are needed to optimize the benefit for this patient group.

Given the contrivance of tumors to continuously evolve, how to select the next line of treatment for EGFRmutant NSCLC progressing on EGFR-TKIs represents a unique and ongoing challenge. It is acknowledged that the approaches should be guided by the identified mechanisms driving resistance whenever possible. Chemotherapy combined with ICIs and anti-angiogenic agents are by far the promising modes for patients, especially with underlying mechanisms unknown. For targetable resistance mechanisms, combining EGFR-TKIs with other oncogene inhibitors, anti-EGFR monoclonal antibodies and systemic treatments may be the better directions of efforts. Besides, the arrival of efficient newer generation of EGFR-TKIs could also shed light on overcoming resistance, but more clinical trials are needed to evaluate their efficacy and safety profiles. In general, despite a number of options already available, additional research and drug development are required to more clearly delineate the treatment paradigms for EGFR-mutant lung cancers after EGFR-TKIs resistance $(27,28)$.

Another interesting finding in this study was that platelet count could be a potential prognostic factor for EGFRmutant advanced NSCLC patients after TKI resistance. Similarly, in a cohort of EGFR-positive NSCLC patients, $\mathrm{Xu}$ et al. (29) found that a decreased platelet count after two cycles of EGFR-TKI treatment was independently correlated with a longer OS ( $\mathrm{P}=0.017, \mathrm{HR}=0.293,95 \% \mathrm{CI}$ : $0.107-0.799)$. In general, paraneoplastic thrombocytosis is associated with many solid tumors and is correlated with reduced survival. Platelets have been associated with various stages of tumor expansion, including local growth, migration in and out of the bloodstream, and metastasis establishment (30). Mechanically, a pathogenic feedback loop may operate between platelets and tumor cells, along with reciprocal interactions between tumor and platelet activation. Tumors can stimulate platelet production and activation through various molecular pathways, and activated platelets can, in turn, promote tumor growth (31). Therefore, an elevated platelet count may imply a dismal prognosis and could potentially serve as a meaningful clinical parameter in establishing a prognostic model for NSCLC patients after disease progression following EGFR-TKI therapy.

Our study demonstrated that both chemo-antiangiogenesis and chemo-immunotherapy combinations could serve as effective salvage therapy options for patients who have failed previous EGFR-TKI treatment. In particular, chemo-antiangiogenesis combinations may be the better choice for T790M-positive patients. This study focused on comparing the efficacy of the two prevalent combination regimens applied in clinical practice, and observing patients with oncogene addiction the chance of ICIs-induced control of disease. Interpretation of our findings needs to take into account that this was a retrospective study without controlled randomization. The lack of consistency in the drugs used for chemotherapy, immunotherapy, and antiangiogenic therapy may also have introduced bias. Furthermore, the overall survival outcomes were premature in both groups up to the data cutoff and warrant further study. Confirmation of these findings will require prospective randomized trials with larger populations.

\section{Conclusions}

In conclusion, this two-center retrospective study showed a comparable efficacy between chemo-antiangiogenesis and chemo-immunotherapy combinations for patients after EGFR-TKI resistance. Chemo-antiangiogenesis combinations may be the preferred option for patients harboring the T790M mutation even after disease progression following osimertinib treatment. Platelet count could be a potential prognostic factor for NSCLC patients after disease progression following EGFR-TKI therapy.

\section{Acknowledgments}

Funding: This study was supported by the Shanghai Municipal Health Commission (grant number: 20164Y0269), the Cancer Research Funding of CSCO- 
Hausen (grant number: Y-HS2019/2-072), the National Natural Science Foundation of China (grant numbers: 81874036, 82072568), the Science and Technology Commission of Shanghai Municipality (grant number: 19411971100) and the Shanghai Shenkang Hospital Development Center (grant number: SHDC12020110).

\section{Footnote}

Reporting Checklist: The authors have completed the STROBE reporting checklist. Available at https://dx.doi. org/10.21037/tlcr-21-681

Data Sharing Statement: Available at https://dx.doi. org/10.21037/tlcr-21-681

Conflicts of Interest: All authors have completed the ICMJE uniform disclosure form (available at https:// dx.doi.org/10.21037/tlcr-21-681). JZ reported that she received funding of Shanghai Municipal Health Commission (20164Y0269). CS reported that she received fundings of the Cancer Research Funding of CSCOHausen (Y-HS2019/2-072), the National Natural Science Foundation of China (81874036, 82072568), the Science and Technology Commission of Shanghai Municipality (19411971100) and the Shanghai Shenkang Hospital Development Center (SHDC12020110). The other authors have no conflicts of interest to declare.

Ethical Statement: The authors are accountable for all aspects of the work in ensuring that questions related to the accuracy or integrity of any part of the work are appropriately investigated and resolved. All procedures performed in this study involving human participants were in accordance with the Declaration of Helsinki (as revised in 2013). The study was approved by the institutional review board of Shanghai Pulmonary Hospital and Shanghai Chest Hospital. Individual consent for this retrospective analysis was waived.

Open Access Statement: This is an Open Access article distributed in accordance with the Creative Commons Attribution-NonCommercial-NoDerivs 4.0 International License (CC BY-NC-ND 4.0), which permits the noncommercial replication and distribution of the article with the strict proviso that no changes or edits are made and the original work is properly cited (including links to both the formal publication through the relevant DOI and the license). See: https://creativecommons.org/licenses/by-nc-nd/4.0/.

\section{References}

1. Mitsudomi T, Suda K, Yatabe Y. Surgery for NSCLC in the era of personalized medicine. Nat Rev Clin Oncol 2013;10:235-44.

2. Herbst RS, Morgensztern D, Boshoff C. The biology and management of non-small cell lung cancer. Nature 2018;553:446-54.

3. Nagano T, Tachihara M, Nishimura Y. Mechanism of Resistance to Epidermal Growth Factor ReceptorTyrosine Kinase Inhibitors and a Potential Treatment Strategy. Cells 2018;7:212.

4. Wang F, Liu X, Bartholdy BA, et al. Blockade of AXL activation overcomes acquired resistance to EGFR tyrosine kinase inhibition in non-small cell lung cancer. Transl Cancer Res 2019;8:2425-38.

5. Chihara Y, Yamada T, Uchino J, et al. Rationale and design of a phase II trial of osimertinib as first-line treatment for elderly patients with epidermal growth factor receptor mutation-positive advanced non-small cell lung cancer (SPIRAL-0 study). Transl Lung Cancer Res 2019;8:1086-90.

6. Leonetti A, Sharma S, Minari R, et al. Resistance mechanisms to osimertinib in EGFR-mutated non-small cell lung cancer. Br J Cancer 2019;121:725-37.

7. Ricordel C, Friboulet L, Facchinetti F, et al. Molecular mechanisms of acquired resistance to third-generation EGFR-TKIs in EGFR T790M-mutant lung cancer. Ann Oncol 2018;29:i28-37.

8. Wu YL, Planchard D, Lu S, et al. Pan-Asian adapted Clinical Practice Guidelines for the management of patients with metastatic non-small-cell lung cancer: a CSCO-ESMO initiative endorsed by JSMO, KSMO, MOS, SSO and TOS. Ann Oncol 2019;30:171-210.

9. Planchard D, Popat S, Kerr K, et al. Metastatic nonsmall cell lung cancer: ESMO Clinical Practice Guidelines for diagnosis, treatment and follow-up. Ann Oncol 2018;29:iv192-iv237. Erratum in: Ann Oncol 2019;30:863-70.

10. Lisberg A, Cummings A, Goldman JW, et al. A Phase II Study of Pembrolizumab in EGFR-Mutant, PD-L1+, Tyrosine Kinase Inhibitor Naïve Patients With Advanced NSCLC. J Thorac Oncol 2018;13:1138-45.

11. Yang JC, Gadgeel SM, Sequist LV, et al. Pembrolizumab in Combination With Erlotinib or Gefitinib as First-Line Therapy for Advanced NSCLC With Sensitizing EGFR Mutation. J Thorac Oncol 2019;14:553-9.

12. Oxnard GR, Yang JC, Yu H, et al. TATTON: a multi-arm, 
phase Ib trial of osimertinib combined with selumetinib, savolitinib, or durvalumab in EGFR-mutant lung cancer. Ann Oncol 2020;31:507-16.

13. Sugiyama E, Togashi Y, Takeuchi Y, et al. Blockade of EGFR improves responsiveness to PD-1 blockade in EGFR-mutated non-small cell lung cancer. Sci Immunol 2020;5:eaav3937.

14. Dong ZY, Zhang JT, Liu SY, et al. EGFR mutation correlates with uninflamed phenotype and weak immunogenicity, causing impaired response to PD-1 blockade in non-small cell lung cancer. Oncoimmunology 2017;6:e1356145.

15. Reck M, Mok TSK, Nishio M, et al. Atezolizumab plus bevacizumab and chemotherapy in non-small-cell lung cancer (IMpower150): key subgroup analyses of patients with EGFR mutations or baseline liver metastases in a randomised, open-label phase 3 trial. Lancet Respir Med 2019;7:387-401.

16. Zhang J, Zhou C, Zhao Y, et al. A PII study of toripalimab, a PD-1 mAb, in combination with chemotherapy in EGFR plus advanced NSCLC patients failed to prior EGFR TKI therapies. J Thorac Oncol 2019;14S:S292.

17. Yang L, Hao X, Hu X, et al. Superior efficacy of immunotherapy-based combinations over monotherapy for EGFR-mutant non-small cell lung cancer acquired resistance to EGFR-TKIs. Thorac Cancer 2020;11:3501-9.

18. Camp RL, Dolled-Filhart M, Rimm DL. X-tile: a new bio-informatics tool for biomarker assessment and outcome-based cut-point optimization. Clin Cancer Res 2004;10:7252-9.

19. Mok TS, Wu YL, Ahn MJ, et al. Osimertinib or PlatinumPemetrexed in EGFR T790M-Positive Lung Cancer. N Engl J Med 2017;376:629-40.

20. Soria JC, Wu YL, Nakagawa K, et al. Gefitinib plus chemotherapy versus placebo plus chemotherapy in EGFR-mutation-positive non-small-cell lung cancer after progression on first-line gefitinib (IMPRESS): a phase 3 randomised trial. Lancet Oncol 2015;16:990-8.

21. Haratani K, Hayashi H, Tanaka T, et al. Tumor immune microenvironment and nivolumab efficacy in EGFR mutation-positive non-small-cell lung cancer based on T790M status after disease progression during EGFRTKI treatment. Ann Oncol 2017;28:1532-9.

22. Hastings K, Yu HA, Wei W, et al. EGFR mutation subtypes and response to immune checkpoint blockade treatment in non-small-cell lung cancer. Ann Oncol 2019;30:1311-20.

23. Yamada T, Hirai S, Katayama Y, et al. Retrospective efficacy analysis of immune checkpoint inhibitors in patients with EGFR-mutated non-small cell lung cancer. Cancer Med 2019;8:1521-9.

24. Ichihara E, Harada D, Inoue K, et al. Characteristics of patients with EGFR-mutant non-small-cell lung cancer who benefited from immune checkpoint inhibitors. Cancer Immunol Immunother 2021;70:101-6.

25. Su S, Dong ZY, Xie Z, et al. Strong Programmed Death Ligand 1 Expression Predicts Poor Response and De Novo Resistance to EGFR Tyrosine Kinase Inhibitors Among NSCLC Patients With EGFR Mutation. J Thorac Oncol 2018;13:1668-75.

26. Garassino MC, Gelibter AJ, Grossi F, et al. Italian Nivolumab Expanded Access Program in Nonsquamous Non-Small Cell Lung Cancer Patients: Results in NeverSmokers and EGFR-Mutant Patients. J Thorac Oncol 2018;13:1146-55.

27. Wu L, Ke L, Zhang Z, et al. Development of EGFR TKIs and Options to Manage Resistance of ThirdGeneration EGFR TKI Osimertinib: Conventional Ways and Immune Checkpoint Inhibitors. Front Oncol 2020;10:602762.

28. Lim SM, Syn NL, Cho BC, et al. Acquired resistance to EGFR targeted therapy in non-small cell lung cancer: Mechanisms and therapeutic strategies. Cancer Treat Rev 2018;65:1-10.

29. Xu L, Xu F, Kong H, et al. Effects of reduced platelet count on the prognosis for patients with non-small cell lung cancer treated with EGFR-TKI: a retrospective study. BMC Cancer 2020;20:1152.

30. Schmied L, Höglund P, Meinke S. Platelet-Mediated Protection of Cancer Cells From Immune Surveillance Possible Implications for Cancer Immunotherapy. Front Immunol 2021;12:640578.

31. Lin RJ, Afshar-Kharghan V, Schafer AI. Paraneoplastic thrombocytosis: the secrets of tumor self-promotion. Blood 2014;124:184-7.

(English Language Editor: D. Fitzgerald)

Cite this article as: $\mathrm{Yu} \mathrm{X}, \mathrm{Li} \mathrm{J}$, Ye L, Zhao J, Xie M, Zhou J, Shen Y, Zhou F, Wu Y, Han C, Qian J, Chu T, Su C. Realworld outcomes of chemo-antiangiogenesis versus chemoimmunotherapy combinations in EGFR-mutant advanced non-small cell lung cancer patients after failure of EGFR-TKI therapy. Transl Lung Cancer Res 2021;10(9):3782-3792. doi: $10.21037 /$ tlcr-21-681 


\section{Supplementary}

Table S1 Univariate and multivariate analyses of clinical parameters for PFS of chemo-antiangiogenesis combinations after TKI resistance (n=100)

\begin{tabular}{|c|c|c|c|c|c|c|}
\hline Factors & \multicolumn{3}{|c|}{ Univariate analysis } & \multicolumn{3}{|c|}{ Multivariate analysis } \\
\hline Age ( $\leq 58 />58$ years) & 1.668 & $1.058-2.628$ & 0.0215 & 1.428 & $0.841-2.426$ & 0.187 \\
\hline Gender (male/female) & 0.6625 & $0.4216-1.041$ & 0.0589 & 1.609 & $0.921-2.811$ & 0.095 \\
\hline Smoking status (current or former/never) & 0.9485 & $0.5630-1.598$ & 0.8431 & & & \\
\hline Median PFS of TKIs ${ }^{a}(\leq 3.1 />3.1$ months) & 2.178 & $1.008-4.706$ & 0.0057 & 2.040 & $0.947-4.395$ & 0.069 \\
\hline $\begin{array}{l}\text { T790M status after TKIs resistance (positive/ } \\
\text { negative) }\end{array}$ & 1.459 & $0.7369-2.890$ & 0.2022 & & & \\
\hline PD-L1 status $(\geq 1 \% /<1 \%)$ & 0.6667 & $0.0902-4.928$ & 0.6419 & & & \\
\hline Neutrophil count $^{\mathrm{a}}\left(\leq 6.99 />6.99 \times 10^{9} / \mathrm{L}\right)$ & 0.4176 & $0.1442-1.210$ & 0.0144 & 1.193 & $0.418-3.406$ & 0.742 \\
\hline Monocyte count ${ }^{\mathrm{a}}\left(\leq 0.68 />0.68 \times 10^{9} / \mathrm{L}\right)$ & 0.5632 & $0.2617-1.212$ & 0.0602 & 0.738 & $0.344-1.583$ & 0.345 \\
\hline Eosinophil count ${ }^{\mathrm{a}}\left(\leq 0.09 />0.09 \times 10^{9} / \mathrm{L}\right)$ & 1.610 & $0.9405-2.755$ & 0.0479 & 1.280 & $0.731-2.243$ & 0.388 \\
\hline Basophil count $^{\mathrm{a}}\left(\leq 0.03 />0.03 \times 10^{9} / L\right)$ & 1.255 & $0.7323-2.152$ & 0.4217 & & & \\
\hline Platelet count ${ }^{a}\left(\leq 373 />373 \times 10^{9} / L\right)$ & 0.5752 & $0.2689-1.230$ & 0.0698 & 0.334 & $0.119-0.937$ & 0.037 \\
\hline $\operatorname{NLR}^{\mathrm{a}}(\leq 1.82 />1.82)$ & 0.5319 & $0.2625-1.078$ & 0.1611 & & & \\
\hline $\operatorname{MLR}^{\mathrm{a}}(\leq 0.26 />0.26)$ & 1.215 & $0.7322-2.016$ & 0.4224 & & & \\
\hline
\end{tabular}

${ }^{a}$, the assessment of the optimal cutoff values were conducted by X-tile software. EGFR, epidermal growth factor receptor; TKIs, tyrosine kinase inhibitors; PD-L1, programmed cell death ligand 1; PFS, progression-free survival; NLR, neutrophil-to-lymphocyte ratio; MLR, monocyte-to-lymphocyte ratio; PLR, platelet-to-lymphocyte ratio. 
Table S2 Univariate and multivariate analyses of clinical parameters for PFS of chemo-immunotherapy combinations after TKI resistance (n=44)

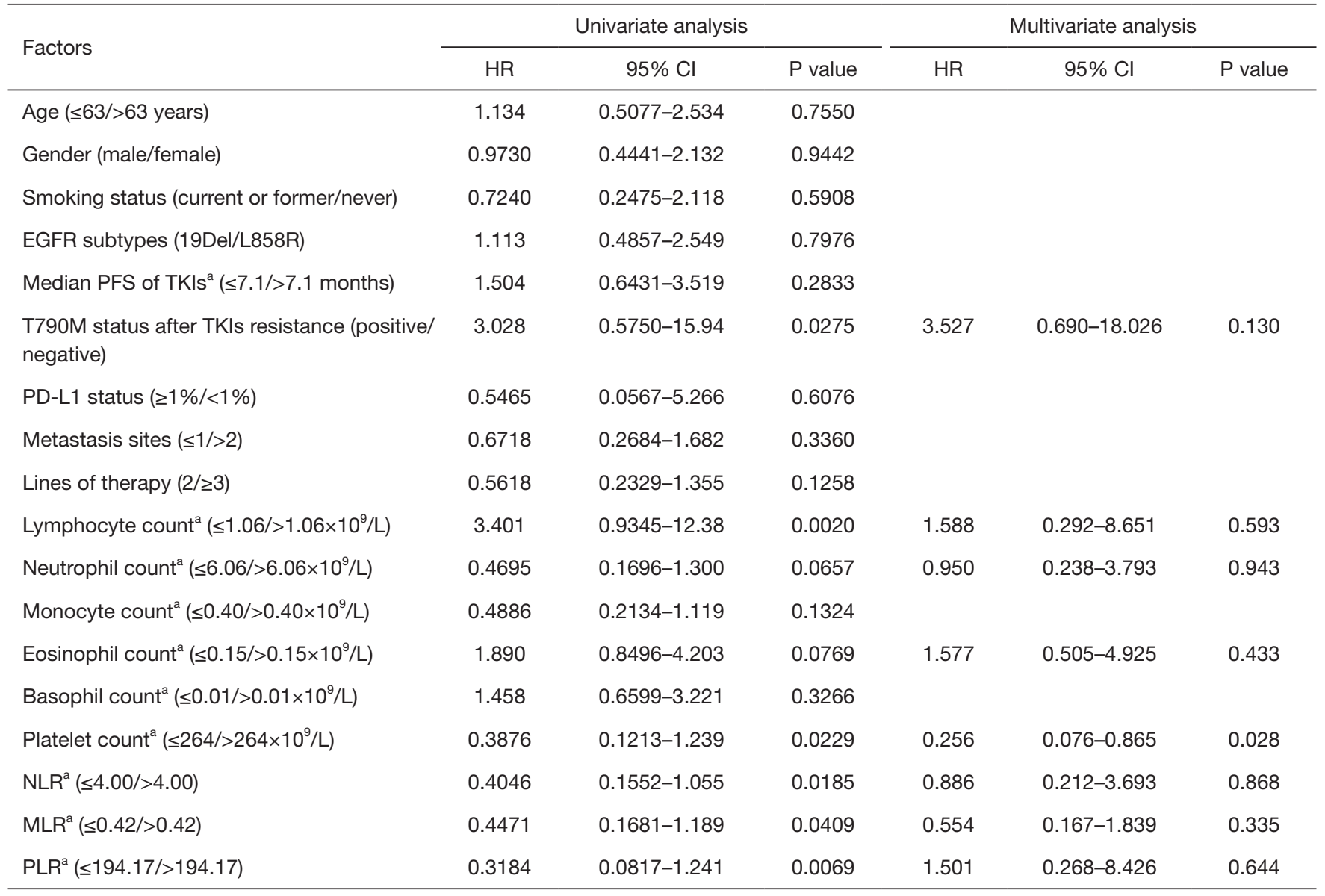

${ }^{\mathrm{a}}$, the assessment of the optimal cutoff values were conducted by X-tile software. EGFR, epidermal growth factor receptor; TKIs, tyrosine kinase inhibitors; PD-L1, programmed cell death ligand 1; PFS, progression-free survival; NLR, neutrophil-to-lymphocyte ratio; MLR, monocyte-to-lymphocyte ratio; PLR, platelet-to-lymphocyte ratio. 